\title{
Molecular Weight Effect on the High Pressure Crystallization of Polyethylene
}

\author{
Munehisa Yasuniwa, Shinsuke Tsubakihara, \\ and Chitoshi NAKAFUKU* \\ Department of Applied Physics, Faculty of Science, \\ Fukuoka University, Jonan-ku, Fukuoka 814-01, Japan \\ * Faculty of Education, Kochi University, Kochi 780, Japan
}

(Received March 30, 1988)

\begin{abstract}
The molecular weight effect on the morphology of high molecular weight polyethylenes (HMW-PE, $2.2 \times 10^{5}$ and $6.9 \times 10^{5}$ ) crystallized at $590 \mathrm{MPa}$ was studied by scanning electron microscopy. The external shape of the band of the HMW-PE is as that of the medium molecular weight PE (MMW-PE); however with increasing molecular weight the band comes to be comprized of long fibrils. It is suggested that the band of HMW-PE is formed by the parallel arrangement of fibrils. Thermal analysis on the pressure-crystallized PEs in a molecular weight range from $4 \times 10^{3}$ to $2.5 \times 10^{6}$ was also performed, and the molecular weight dependence of the melting temperature and heat of fusion was obtained. The heat of fusion of the pressure-crystallized sample decreases linearly with logarithm of molecular weight. The melting temperature increases linearly with logarithm of molecular weight in a molecular weight region lower than about $10^{5}$. Above $10^{5}$, the melting temperature for the pressure-crystallized powder sample is higher than about $146^{\circ} \mathrm{C}$ and increases slightly with molecular weight. This considerable increase of the melting temperature is caused by the superheating effect.
\end{abstract}

KEY WORDS Polyethylene / Molecular Weight / High Pressure /

Crystallization / Melting / Morphology / Fibril / SEM / DSC /

High pressure crystallization of polyethylene (PE) has been studied in the past two decades. The growth of the so-called "extendedchain crystal" (ECC) and the formation of the band for a pressure-crystallized medium molecular weight PE (MMW-PE) have been reported. $^{1-5}$ It seems to be commonly accepted that the band is formed by the growth of ECC. The detailed formation mechanism of the band for MMW-PE has been suggested by Asai et al. ${ }^{6,7}$

In contrast, a strikingly different morphology has been reported recently by Yasuniwa and $\mathrm{Nakafuku}^{8}$ for a pressurecrystallized ultra-high molecular weight PE (UHMW-PE). Fibrous bands of long fibrils and bands of thick fibrils closely spaced were observed by electron microscopy for pressure- crystallized as-polymerized (powder) and bulk samples, respectively. Further, it has been suggested that the band of UHMW-PE is composed of fibrils with parallel arrangement, and that the basic structure of the band is formed in the molten state.

These studies indicate that a drastic change of the morphology of the band occurs in the intermediate region between UHMW and MMW, i.e., high molecular weight (HMW) region, and that the formation mechanism of the band changes in the HMW region. The morphology and the band formation mechanism for the pressure-crystallized PE in the HMW region are the points of emphasis in the present investigation.

It is reasonable to consider that the lamellar thickness and crystallinity, which can be esti- 
mated from the melting temperature and the heat of fusion, should change with molecular weight on the same crystallization condition. Molecular weight effect on the melting temperature and the heat of fusion for the pressure-crystallized $\mathrm{PE}$ ranging from MMW to UHMW has been reported by Hoehn et $a l .^{9,10}$ However, the consistent molecular weight dependence of the melting temperature and heat of fusion cannot be obtained from their data.

In the present paper, a detailed morphology of the pressure-crystallized HMW-PE is investigated to study the effect of molecular weight on the formation of the band. In addition, molecular weight effect on the melting temperature and heat of fusion for pressurecrystallized PE ranging from low molecular weight (LMW) to UHMW is determined.

\section{EXPERIMENTAL}

Unfractionated high density PE samples with different molecular weights were used in this study. Viscosity averaged molecular weights of the samples are summarized in Table I. Samples $1-3$ were supplied by Mitsui Petrochemical Industries Co. The others (samples 4 -6) were supplied by Showa Denko Co. These samples are classified into four groups according to molecular weight. Sample 1 , samples 2 and 3, samples 4 and 5 , and sample 6 are classified into low-, medium-, high-, and ultra-high molecular weight $\mathrm{PE}$ (LMW-, MMW-, HMW-, and UHMW-PE), respectively.

Two types of samples, powder and bulk, were used in this experiment. The powder samples were as-polymerized resins. The bulk samples were melt-crystallized (kneaded) ones. The detailed procedure for the preparation of the bulk sample is mentioned in reference 11 .

High pressure crystallization was carried out by the use of high pressure DTA apparatus described elsewhere. ${ }^{12}$ The heating and cooling rates were precisely controlled by a pro-
Table I. Molecular weight (viscosity-averaged) of the samples

\begin{tabular}{ccl}
\hline Sample No. & Molecular weight & Group \\
\hline 1 & $4 \times 10^{3}$ & LMW \\
2 & $1 \times 10^{4}$ & MMW \\
3 & $6.7 \times 10^{4}$ & MMW \\
4 & $2.2 \times 10^{5}$ & HMW \\
5 & $6.9 \times 10^{5}$ & HMW \\
6 & $2.5 \times 10^{6}$ & UHMW \\
\hline
\end{tabular}

grammable automatic temperature controller (Sinku-Riko Co. Model HPC-7105). The hydrostatic pressure was measured within \pm 1 $\mathrm{MPa}$ by a Bourdon gauge (Heise Co.) connected to the high pressure vessel. Low viscosity silicone oil (Shin'etsu KF-96, 0.1 St) was used as the pressure-transmitting fluid.

The powder sample was prepared in the form of a rod ( $c a .1 .2 \mathrm{~mm}$ in diameter and $10 \mathrm{~mm}$ in length) by compressing the original powder sample in a piston cylinder type cell at room temperature. The bulk sample was prepared in the same shape as the rod powder sample by shaving the original bulk sample with a razor blade. In high pressure crystallization samples were coated by epoxy resin to avoid the effect of the pressure-transmitting fluid.

High pressure crystallization of these samples was performed as follows on the basis of the DTA results in the previous paper. ${ }^{11} \mathrm{At}$ $590 \mathrm{MPa}$ the sample was heated up to a temperature $10 \mathrm{~K}$ higher than the end of the DTA melting peak of the high pressure phase (hexagonal phase) at $5 \mathrm{~K} \mathrm{~min}^{-1}$ and held at the temperature (molten state) for two minutes. It was then cooled to a temperature lower than $100^{\circ} \mathrm{C}$ at $2 \mathrm{~K} \mathrm{~min}^{-1}$ under the same pressure.

Specimens for electron microscopy were prepared by brittle fracturing the rod samples in liquid nitrogen. The fracture surface was coated with $\mathrm{Au}$ and examined with a Hitachi S-430 SEM at magnifications up to $30,000 \times$. For high magnification (up to $50,000 \times$ ), electron micrographs were taken with a Hitachi 
S-800 field emission type SEM.

Differential scanning calorimetry (DSC) was performed at $4 \mathrm{~K} \mathrm{~min}{ }^{-1}$ with a Rigaku Denki low temperature thermal analyzer. Indium was used as the calibration standard.

\section{RESULTS AND DISCUSSION}

The scanning electron micrographs for the pressure-crystallized powder and bulk samples of HMW-PE (MW $2.2 \times 10^{5}$ ) are shown in Figures 1 and 2, respectively. In contrast to the case of UHMW-PE, ${ }^{7}$ there is no distinct difference in the morphology between the pressure-crystallized powder and bulk samples of HMW-PE.

As shown in Figure 1, the external shape of the band resembles that of a pressurecrystallized MMW-PE which has been reported by many authors. However, there is a clear difference in the band between the pressure-crystallized HMW and MMW samples. The microscopic difference is that some part of the band in HMW sample is formed by fibrils. There are many long fibrils whose width is approximately $50 \mathrm{~nm}$, and some long fibrils are included in the band as shown by the arrow in Figure 2. The axis of the long fibril on the surface of the band is parallel to the striation in the band.

The band for a pressure-crystallized MMWPE (ordinary band) results from the crystallization of molecular chains. In contrast, the fibrous band for the pressure-crystallized UHMW-PE results from the parallel arrangement of fibrils. ${ }^{8}$ As shown in Figures 1 and 2, the band for the pressure-crystallized HMW sample consists of an ordinary band and a fibrous band. This indicates that the band for the pressure-crystallized HMW-PE is formed by crystallization of the molecular chains and parallel arrangement of the fibrils.

Macroscopic differences in the band between the pressure-crystallized MMW and HMW samples are as follows. Bands in the HMW sample are not spaced closely. There

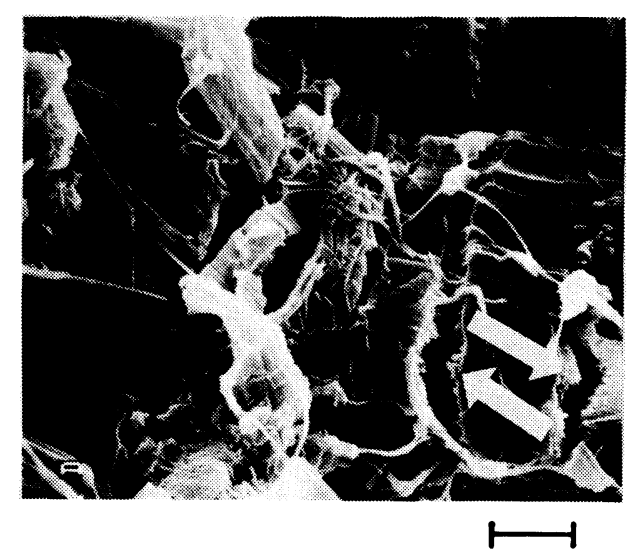

Figure 1. SEM photograph of the fracture surface for the pressure-crystallized powder sample of HMW-PE (MW $2.2 \times 10^{5}$ ). The sample was obtained by crystallization of the powder sample at a cooling rate of $2 \mathrm{~K} \mathrm{~min}^{-1}$ at $590 \mathrm{MPa}$ from the melt. The scale bar represents $1.0 \mu \mathrm{m}$. The explanations on the arrows drawn in the photograph are given in the text.

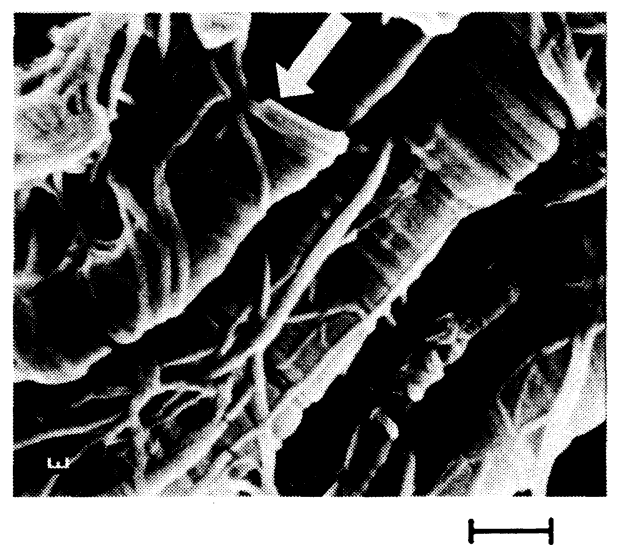

Figure 2. SEM photograph of the fracture surface for the pressure-crystallized bulk sample of HMW-PE (MW $\left.2.2 \times 10^{5}\right)$. The scale bar represents $0.3 \mu \mathrm{m}$. The explanation on the arrow drawn is given in the text.

are many cracks between the bands as shown in Figures 1 and 2, contrary to the closely spaced band appearing usually in the pressurecrystallized MMW-PE. As shown by the arrows in Figure 1, the concave portion on the side surface fits the convex portion on the oppsoite side surface. This fact indicates that the cracking between the bands is caused at the boundary of the bands. The crack may be 
formed in the releasing process of pressure and/or in the fracturing process of the sample.

Scanning electron micrographs of the fracture surface of the pressure-crystallized powder and bulk samples of HMW-PE (MW $6.9 \times 10^{5}$ ) are shown in Figure 3 and Figure 4, respectively. The quantity of the fibrils and spaces between bands in these samples increased compared with those of MW $2.2 \times 10^{5}$. That is, the band comes to be comprized of long fibrils with increasing molecular weight. Though the direction of the long axis of the fibril in the band is disarranged, the external shape of the band is not so disordered. This indicates that the external shape of the ordinary band is formed by the parallel arrangement of fibrils even in this molecular weight region.

The morphology for the pressure-crystallized UHMW powder sample is distinctly different from that of the bulk sample. ${ }^{8}$ The former shows fibrous band, and the latter shows textile structure. The morphologies of powder and bulk samples for MW of $6.9 \times 10^{5}$ are intermediate between those of UHMW and the ordinary band, as shown in Figures 3 and 4. There is a slight difference in the morphology between the powder and bulk samples, suggesting that the original form of the sample (bulk or powder) affects the morphology of the pressure-crystallized sample in the molecular weight region higher than HMW.

Morphologies of the pressure-crystallized HMW-PE have been reported by some authors. ${ }^{9,13,14}$ However they have neither elucidated the detailed structure of the band nor suggested that the band is formed by fibrils.

Since the pressure-crystallized sample was heated over the melting temperature under high pressure, crystallites in the fibrils should be melted. However, the shape of the fibrils in the pressure-crystallized HMW and UHMW samples hardly changed. The explanation for this is that the remarkable disentangling between molecular chains in the fibril does not

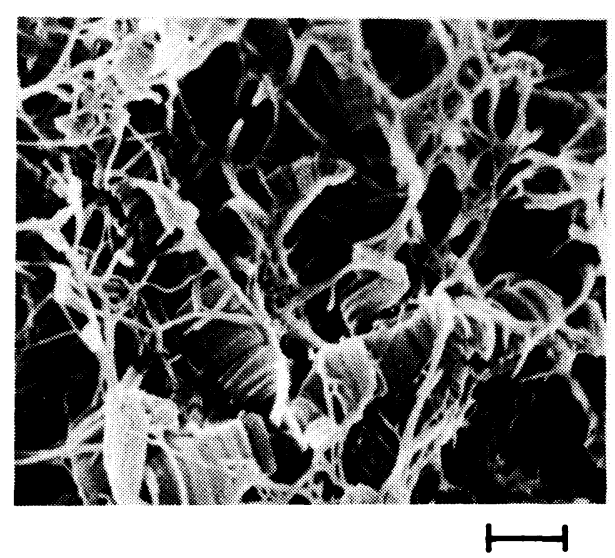

Figure 3. SEM photograph of the fracture surface for the pressure-crystallized powder sample of HMW-PE (MW $6.9 \times 10^{5}$ ). The scale bar represents $1.0 \mu \mathrm{m}$.

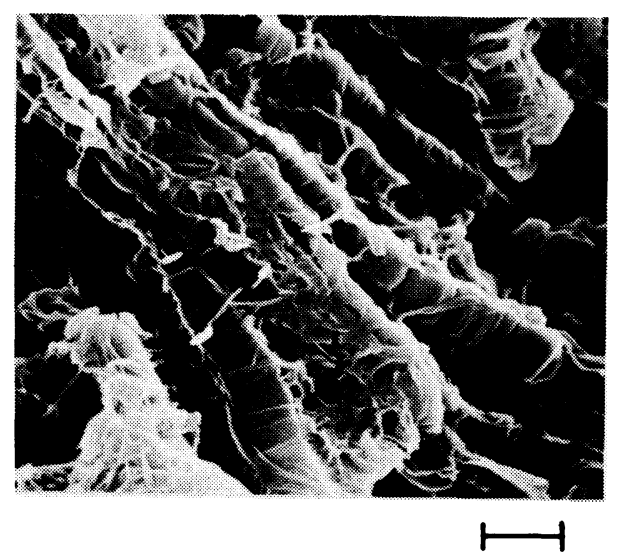

Figure 4. SEM photograph of the fracture surface for the pressure-crystallized bulk sample of HMW-PE (MW $\left.6.9 \times 10^{5}\right)$. The scale bar represents $1.0 \mu \mathrm{m}$.

occur during crystallization. Therefore the crystallinity and the crystallite size in the fibril are considered to be low and samll, respectively. As the quantity of the fibrils increases and the band becomes irregular in pressurecrystallized sample with increasing molecular weight, the crystallinity and the lamellar thickness become low and small.

DSC melting curves for the pressurecrystallized powder and bulk samples are shown in Figures 5(a) and (b), respectively. The DSC curves of PE with molecular weight 
(a)

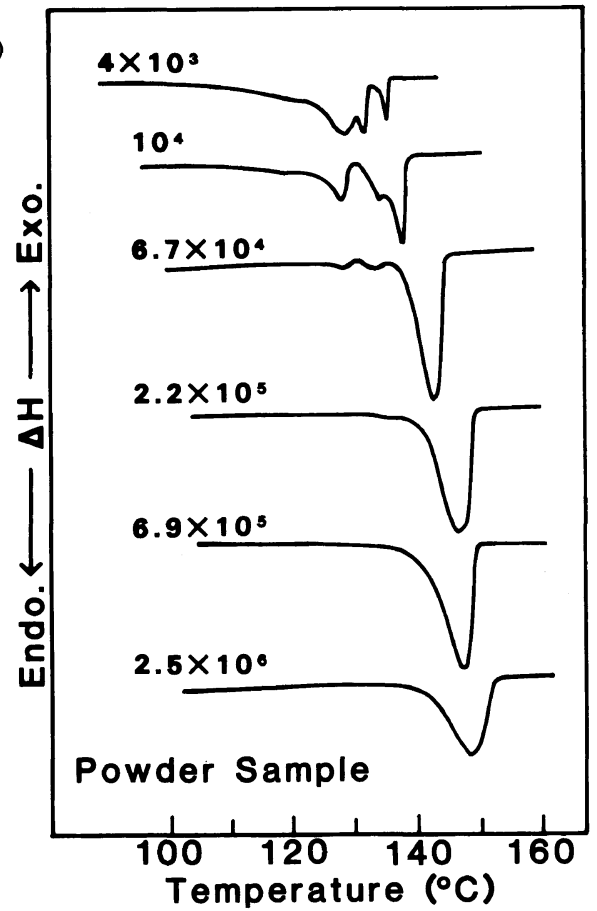

(b)

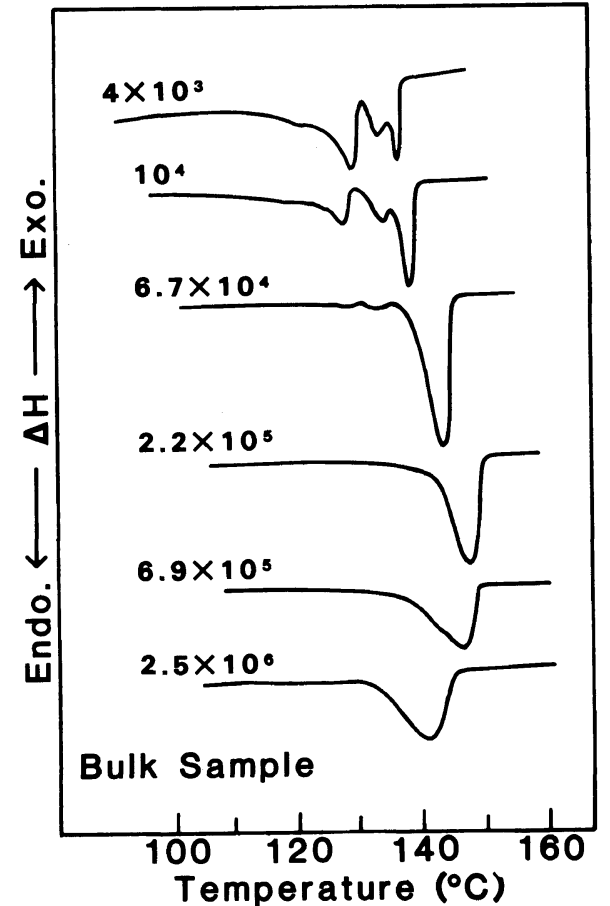

Figure 5. Molecular weight effect on the DSC melting curves for the pressure-crystallized samples. (a) pressurecrystallized powder sample; (b) pressure-crystallized bulk sample.
Table II. Melting peak temperatures and heats of fusion for the pressure-crystallized powder and bulk samples. $T_{\mathrm{p}}$, peak temperature of DSC curve; $\Delta H$, heat of fusion calculated from the area of DSC melting curve

\begin{tabular}{|c|c|c|c|c|}
\hline \multirow{3}{*}{$\begin{array}{c}\text { Sample } \\
\text { No. }\end{array}$} & \multicolumn{2}{|c|}{ Powder } & \multicolumn{2}{|c|}{ Bulk } \\
\hline & $T_{\mathrm{p}}$ & $\Delta H$ & $T_{\mathrm{p}}$ & $\Delta H$ \\
\hline & ${ }^{\circ} \mathrm{C}$ & cal g $^{-1}$ & ${ }^{\circ} \mathrm{C}$ & $\mathrm{calg}^{-1}$ \\
\hline 1 & 134.7 & - & 135.2 & - \\
\hline 2 & 136.9 & - & 137.2 & - \\
\hline 3 & 142.0 & 66.4 & 142.0 & 67.5 \\
\hline 4 & 145.7 & 63.3 & 146.3 & 59.9 \\
\hline 5 & 146.2 & 60.5 & 145.3 & 56.1 \\
\hline 6 & 147.5 & 54.0 & 141.7 & 49.1 \\
\hline
\end{tabular}

of $4 \times 10^{3}, 10^{4}$, and $6.7 \times 10^{4}$ show three peaks. The highest peak corresponds to the melting of the thickest lamellae, that is ECC. The origin of the other peaks has not been determined.

Melting peak temperatures and heat of fusions calculated from the areas of the peaks are listed in Table II. The listed melting temperatures correspond to the highest peaks among DSC melting peaks. The heats of fusion of molecular weights $4 \times 10^{3}$ and $10^{4}$ were not obtained, because the area of the highest melting temperature peak is considerably smaller than the integrated area of the residual peaks, as shown in Figure 5. The listed data for pressure-crystallized powder and bulk samples of molecular weight $2.5 \times 10^{6}$ are averages obtained by the present and previous DSC measurements. ${ }^{7}$ The listed data almost coincide with the previous ones within experimental error, which is supposed to be mainly caused by broadness of the DSC melting peaks.

Figures 6 and 7 show molecular weight dependence of the heat of fusion and melting peak temperature for pressure-crystallized PE, respectively. As shown in Figure 6, the decrease of the heats of fusion of the pressurecrystallized powder and bulk samples is approximately linear with a logarithm of molecular weight. The decrease of the heat of fusion of 
the pressure-crystallized bulk sample is larger than that of the pressure-crystallized powder sample. The heat of fusion of the equilibrium crystal of PE is about $70 \mathrm{calg}^{-1} \cdot{ }^{15}$ Those of the pressure-crystallized powder and bulk samples in the UHMW region are considerably lower than the value of the equilibrium crystal as shown in Figure 6 and Table II, which can be explained by the following mechanism in-

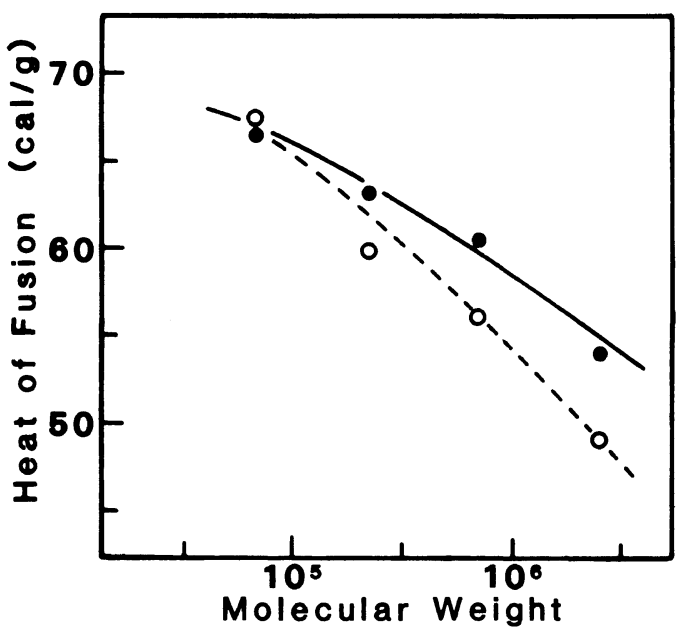

Figure 6. Molecular weight effect on the heat of fusion of the pressure-crystallized samples. crystallized powder sample; $\bigcirc$, pressure-crystallized bulk sample. volving entanglements; the substantial features have been mentioned in ref 8 and 11 .

Smith et al. ${ }^{16,17}$ have suggested that for the quenched UHMW-PE, all entanglements existing in the melt are trapped during solidification process. As the molecular chain length of HMW- and UHMW-PE is approximately the same as the width of a band $(c a .1 \mu \mathrm{m})$ or longer than it, during crystallization under high pressure the chain disentangling would be difficult to occur on the time scale of the present experimental condition, and therefore the entanglements should be trapped in the sample. The entanglements hinder the crystal growth and bring defects in the crystal. Therefore crystals become smaller and more disordered with molecular weight in the HMW and UHMW region, resulting in its decrease in the heat of fusion.

It has been indicated that entanglements are introduced by the mechanical process in the preparation of a bulk sample and tend to be trapped in a UHMW bulk sample; the pressure-crystallized bulk sample contains more entanglements than the pressurecrystallized powder sample. ${ }^{10}$ The considerable decrease of heat of fusion for the pressurecrystallized bulk sample in HMW and

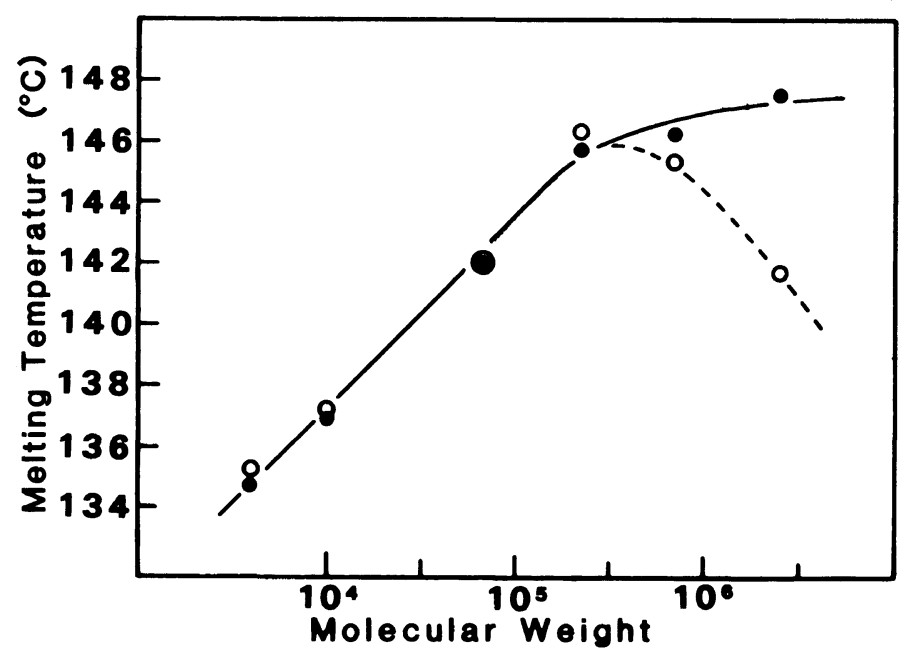

Figure 7. Molecular weight effect on the peak melting temperature of the pressure-crystallized samples. - pressure-crystallized powder sample; $O$, pressure-crystallized bulk sample. 
UHMW regions can be explained by the entanglements introduced.

As shown in Figure 7, the melting temperature increases linearly with logarithm of molecular weight in a molecular weight region lower than about $10^{5}$. Above $10^{5}$, as in the case of HMW and UHMW regions, the increasing rate of the melting temperature is small, and the melting temperature is higher than about $146^{\circ} \mathrm{C}$ which is considerably higher than the reported equilibrium melting temperature of PE $\left(141^{\circ} \mathrm{C}\right){ }^{15}$ It is well known that the superheating in the melting of the sample becomes remarkable with increasing molecular weight. $^{4}$

Melting temperature of PE lamellar crystals as a function of the reciprocal lamellar thickness is expressed by the equation $(1)^{15}$

$$
T_{\mathrm{m}}=414.2[1-(6.27 / l)] \pm 0.8 \mathrm{~K}
$$

where $l$ is lamellar thickness. If the lamellar crystal is formed by fully extended-chain, the lamellar thickness is equivalent to the chain length. Since molecular weight of 28.05 corresponds to chain length of $2.546 \AA$ in PE chain, the above equation can be expressed as follows.

$$
T_{\mathrm{m}}=414.2[1-(69.1 / \mathrm{MW})] \pm 0.8 \mathrm{~K}
$$

where $\mathrm{MW}$ is molecular weight.

Figure 8 shows the peak melting temperature of the pressure-crystallized powder sample as a function of the reciprocal chain length and molecular weight. The dotted line and the hatched area show the above equation. As mentioned above, the entanglement is considered to increase with molecular weight. Since the increase of entanglements brings many small and disordered crystals, the melting temperature of these crystals decreases with increasing entanglements. This entanglement effect is remarkable on change of the melting temperature in HMW and UHMW regions. On the contrary, the melting temperature increases by superheating which is also remarkable in HMW and UHMW regions. ${ }^{4}$

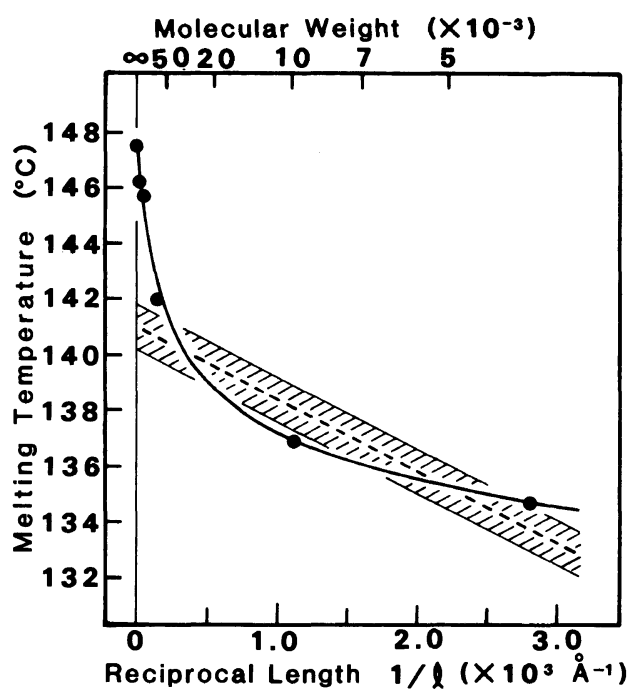

Figure 8. Peak melting temperatures of the pressurecrystallized powder sample for the reciprocal chain length. The dotted line and the hatched area are expressed by the equation (1).

As shown in Figure 8, the melting temperature is increased by superheating abruptly with decreasing reciprocal chain length, i.e., with increasing molecular weight, in the HMW and UHMW regions.

The decrease of the melting temperature for pressure-crystallized bulk sample in UHMW region is explained as the result of competition of the two effects of entanglement. The linear increase of the melting temperature in the LMW region indicates the formation of fully extended-chain crystals. In the MMW and HMW region, these two effects should contribute to change the melting temperature.

A consistent molecular weight dependence of melting temperature in the molecular weight range from about $10^{3}$ to $10^{6}$ has been reported for linear PE fractions and paraffins by Mathot and Pijpers. ${ }^{18}$ Their sample was not ECC but a folded-chain crystal in which lamellar thickening perhaps occurred in the heating process, especially near the melting temperature. On the other hand, Hoehn et al. have presented heats of fusion and melting tempera- 
tures obtained for ECC samples in the molecular weight range from $4.9 \times 10^{4}$ to $4.6 \times 10^{6}$. However, consistent molecular weight dependence of the heat of fusion and melting temperature was not recognized from their data. The differences between their and the present data are supposed to be caused from the differences of the sample preparation procedure and heating rate of DSC.

Acknowledgments. The authors would like to thank Hitachi, Ltd. for taking high magnification electron micrographs with a field emission type SEM (S-800). They are also indebted to Showa Denko Co. and Mitsui Petrochemical Industries Co. for providing experimental resins. This work was partly supported by a grant from the Central Research Institute of Fukuoka University.

\section{REFERENCES}

1. B. Wunderlich and T. Arakawa, J. Polym. Sci., A-2, 7, 3697 (1964).

2. P. H. Geil, F. R. Anderson, B. Wunderlich, and T. Arakawa, J. Polym. Sci., A-2, 7, 3707 (1964).
3. M. Yasuniwa, C. Nakafuku, and T. Takemura, Polym. J., 4, 526 (1973).

4. B. Wunderlich, "Macromolecular Physics," Vol. 1, Academic Press, New York, N. Y., 1973.

5. D. C. Bassett, "Development in Crystalline Polymers," Vol. 1, Applied Science Publishers, Essex, England, 1982, Chapter 3.

6. T. Asahi, Y. Miyamoto, H. Miyaji, and K. Asai, Polymer, 23, 773 (1982).

7. S. Miyashita, T. Asahi, H. Miyaji, and K. Asai, Polymer, 26, 1791 (1985).

8. M. Yasuniwa and C. Nakafuku, Polym. J., 19, 805 (1987).

9. H. H. Hoehn, R. C. Ferguson, and R. R. Hebert, Polym. Eng. Sci., 18, 457 (1978).

10. R. C. Ferguson and H. H. Hoehn, Polym. Eng. Sci., 18, 466 (1978).

11. M. Yasuniwa, K. Haraguchi, C. Nakafuku, and S. Hirakawa, Polym. J., 17, 1209 (1985).

12. N. Hiramatsu and S. Hirakawa, Polym. J., 12, 105 (1980).

13. R. B. Prime and B. Wunderlich, J. Polym. Sci., A-2, 7, 2061 (1969).

14. D. V. Rees and D. C. Bassett, J. Polym. Sci., A-2, 9, 385 (1971).

15. B. Wunderlich, "Macromolecular Physics," Vol. 3, Academic Press, New York, N. Y., 1980, Chapter 8.

16. P. Smith, P. J. Lemstra, and H. C. Booiji, J. Polym. Sci., Polym. Phys. Ed., 19, 877 (1981).

17. P. Smith, Macromolecules, 16, 1802 (1983).

18. V. B. F. Mathot and M. F. J. Pijpers, Polym. Bull., 11, 297 (1984). 